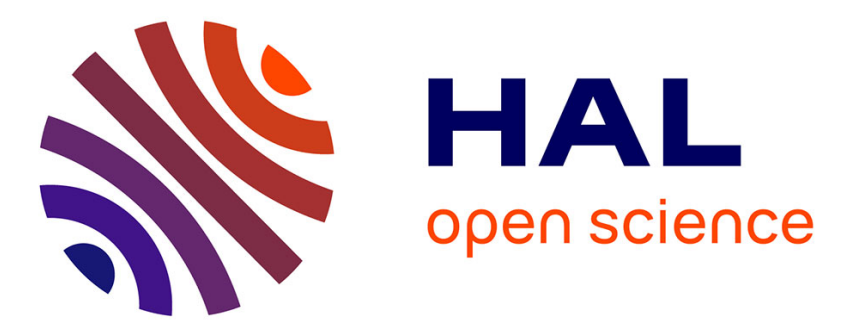

\title{
Charge density wave properties of the quasi two-dimensional purple molybdenum bronze KMo6O17
}

Hafid Balaska, Jean Dumas, Hervé Guyot, P. Mallet, Jacques Marcus, Claire Schlenker, Jean-Yves Veuillen, David Vignolles

\section{- To cite this version:}

Hafid Balaska, Jean Dumas, Hervé Guyot, P. Mallet, Jacques Marcus, et al.. Charge density wave properties of the quasi two-dimensional purple molybdenum bronze KMo6O17. Solid State Sciences, 2005, 7 (6), pp.690-698. 10.1016/j.solidstatesciences.2004.11.015 . hal-00015951

\section{HAL Id: hal-00015951 \\ https://hal.science/hal-00015951}

Submitted on 9 Feb 2022

HAL is a multi-disciplinary open access archive for the deposit and dissemination of scientific research documents, whether they are published or not. The documents may come from teaching and research institutions in France or abroad, or from public or private research centers.
L'archive ouverte pluridisciplinaire HAL, est destinée au dépôt et à la diffusion de documents scientifiques de niveau recherche, publiés ou non, émanant des établissements d'enseignement et de recherche français ou étrangers, des laboratoires publics ou privés.

\section{(c) (1) $\$$}

Distributed under a Creative Commons Attribution - NonCommercial| 4.0 International 


\title{
Charge density wave properties of the quasi two-dimensional purple molybdenum bronze $\mathrm{KMo}_{6} \mathrm{O}_{17}$
}

\author{
H. Balaska ${ }^{\mathrm{a}, 1}$, J. Dumas ${ }^{\mathrm{a}}$, H. Guyot ${ }^{\mathrm{a}}$, P. Mallet ${ }^{\mathrm{a}}$, J. Marcus ${ }^{\mathrm{a}}$, C. Schlenker ${ }^{\mathrm{a}, *}$, J.Y. Veuillen ${ }^{\text {a }}$, \\ D. Vignolles ${ }^{\mathrm{b}}$ \\ a Laboratoire d'Etudes des Propriétés Electroniques des Solides, CNRS, BP 166, 38042 Grenoble Cedex 9, France ${ }^{2}$ \\ ${ }^{\mathrm{b}}$ Laboratoire National des Champs Magnétiques Pulsés, 143 Avenue de Rangueil, 31077 Toulouse, France
}

\begin{abstract}
The purple molybdenum bronze $\mathrm{KMo}_{6} \mathrm{O}_{17}$ is a quasi-two-dimensional compound which shows a Peierls transition towards a commensurate metallic CDW state. Electron spectroscopy (ARUPS), Scanning Tunnelling Microscopy (STM) and spectroscopy (STS) as well as high magnetic field studies are reported. ARUPS studies corroborate the model of the hidden nesting and provide a value of the CDW vector in good agreement with other measurements. STM studies visualize the triple- $q$ CDW in real space. This is consistent with other measurements of the CDW vector. STS studies provide a value of several $10 \mathrm{meV}$ for the average CDW gap. High magnetic field measurements performed in pulsed fields up to $55 \mathrm{~T}$ establish that first order transitions to smaller gap states take place at low temperature. These transitions are ascribed to Pauli type coupling. A phase diagram summarizing all observed anomalies and transitions is presented.
\end{abstract}

Keywords: Charge density waves; Molybdenum bronzes; STM; ARUPS; High magnetic fields

\section{Introduction}

The so-called purple bronze $\mathrm{KMo}_{6} \mathrm{O}_{17}$ belongs to the class of the low dimensional conducting transition metal oxide bronzes which show anisotropic Fermi surfaces and therefore CDW instabilities [1-3]. Among these materials, $\mathrm{KMo}_{6} \mathrm{O}_{17}$ is a quasi two-dimensional (2D) metal due to a trigonal layered structure including $\mathrm{MoO}_{6}$ octahedra layers separated by $\mathrm{K}^{+}$ions and $\mathrm{MoO}_{4}$ tetrahedra [4] (Fig. 1). The conduction electrons are confined in the infinite layers perpendicular to the $c$-axis, leading to quasi-2D electronic prop-

\footnotetext{
${ }^{*}$ Corresponding author. Tel.: +33 (0)4 768810 86; Fax: +33 (0)4 7688 7988.

E-mail address: schlenke@grenoble.cnrs.fr (C. Schlenker).

${ }^{1}$ Permanent address: University of Skikda, Algeria.

2 Associated to Université Joseph Fourier and Institut National Polytechnique de Grenoble.
}

erties. Electrical resistivity and magnetic susceptibility studies, followed by crystallographic investigations, have established that a Peierls instability occurs at $T_{\mathrm{P}}=110 \mathrm{~K}$ towards a metallic commensurate charge density wave state. This is due to openings of partial gaps on the quasi-cylindrical Fermi surface. Band structure calculations with a quasi-twodimensional (2D) tight binding method lead to three partially filled bands crossing the Fermi level [5]. They correspond to three quasi-1D Fermi surface sheets related to zig-zag $\mathrm{MoO}_{6}$ octahedra chains along the $a, b$ and $(a+b)$ directions. This is the so-called hidden nesting property. These calculations predict nesting wave vectors $\left(a^{*} / 2,0,0\right)$ and equivalent, which have been observed by electron diffraction and X-ray diffuse scattering [6].

Band structure studies have been performed by angleresolved photoemission spectroscopy (ARPES) on the parent compound $\mathrm{NaMo}_{6} \mathrm{O}_{17}$ at room temperature [7]. A preliminary report of similar studies on $\mathrm{KMo}_{6} \mathrm{O}_{17}$ has been re- 


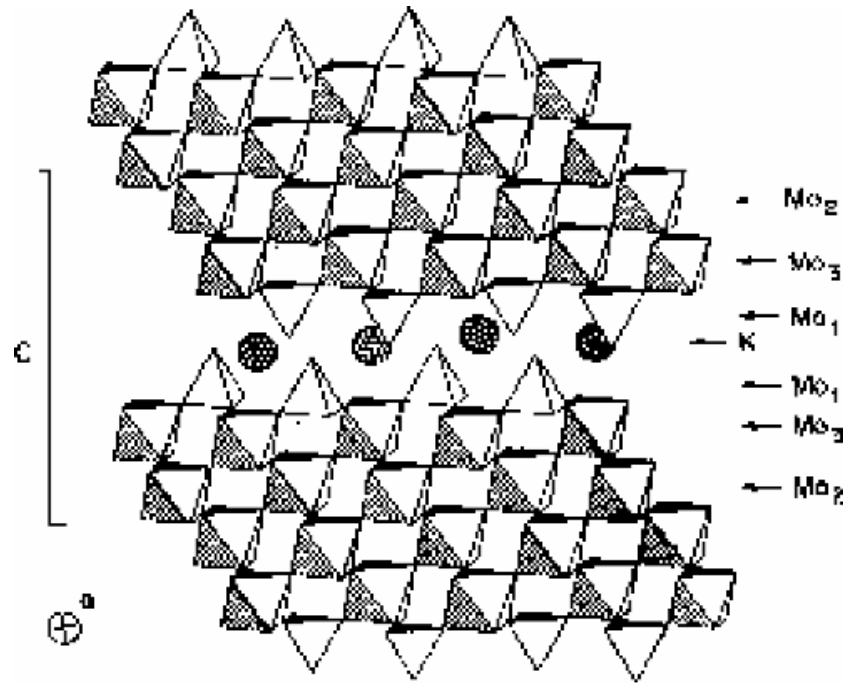

Fig. 1. Crystal structure of $\mathrm{KMo}_{6} \mathrm{O}_{17}$ showing the oxygen octahedra and tetrahedra. View along the $a$-axis. From Ref. [4].

ported elsewhere [8]. Fermi surface imaging performed with the same technique both on $\mathrm{KMo}_{6} \mathrm{O}_{17}$ and on $\mathrm{NaMo}_{6} \mathrm{O}_{17}$ have corroborated the hidden nesting model [7]. All explored physical properties correspond to a CDW gap opening at $110 \mathrm{~K}$ with a doubling of the unit cell in the $(a, b)$ plane. STM studies, reported recently, establish that the superstructure is a triple- $q(2 a \times 2 b)$ hexagonal one [9].

In the CDW state, the in-plane magnetoresistance $\Delta \rho / \rho_{0}$ measured with the magnetic field perpendicular to the layers has been found positive and very large (Fig. 2). This has been attributed to the coexistence of hole and electron pockets with high mobilities on the Fermi surface of the CDW state. High magnetic field studies performed with pulsed fields up to $38 \mathrm{~T}$ showed several anomalies on the curves $\Delta \rho / \rho_{0}$ vs field, followed by an anomalous decrease of the magnetoresistance above $30 \mathrm{~T}$ [10]. A preliminary report of more recent measurements performed up to $55 \mathrm{~T}$

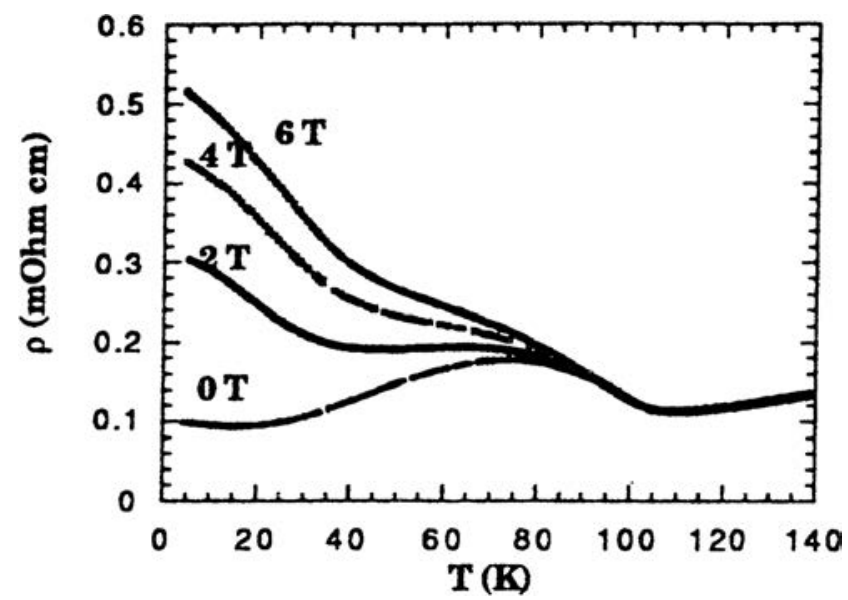

Fig. 2. Resistivity as a function of temperature for different applied magnetic fields perpendicular to the $2 \mathrm{D}$ layers, current in the plane of the layers. shows that a first order transition takes place above $25 \mathrm{~T}$ [11]. Similar large and positive magnetoresistance has also been observed up to $15 \mathrm{~T}$ in the parent compound $\mathrm{TlMo}_{6} \mathrm{O}_{17}$ [12].

We have collected in this review paper experimental data performed on $\mathrm{KMo}_{6} \mathrm{O}_{17}$ in these last years, including ARUPS studies of the band structure, STM imaging and spectroscopy of the CDW state as well as new experimental evidence of a first order magnetic field induced transition at low temperature. These results show that $\mathrm{KMo}_{6} \mathrm{O}_{17}$ is a model quasi two-dimensional compound. The field induced transition is similar to the so-called kink transition found in the quasi-2D organic salts of the (BEDT-TTF $)_{2} \mathrm{X}$ family [13]. This establishes that these phenomena are characteristic of quasi-2D metals and not specific of one type of compounds.

\section{Experimental techniques}

Single crystals used in these studies have been grown by electrolytic reduction of a $\mathrm{K}_{2} \mathrm{CO}_{3}-\mathrm{MoO}_{3}$ melt. They are in shape of small platelets, typically $3 \times 1 \times 0.2 \mathrm{~mm}^{3}$, perpendicular to the $c$-axis [6].

ARUPS measurements have been performed at room temperature, using He-lamp radiations, on (001) surfaces freshly cleaved in UHV. The crystals were oriented using either the natural edges or the LEED patterns (Fig. 3). However the surface of the compound is unstable to electron bombardment and the LEED patterns are obtained for a short time only.

Measurements of the local density of states (LDOS) in the normal and CDW phase have been performed using a home-made Variable Temperature Scanning Tunnelling Microscope (VT-STM), described in reference [9]. The sample was cleaved in UHV ( $\left.p=1.0 \times 10^{-10} \mathrm{mb}\right)$ and STM measurements were made either at $300 \mathrm{~K}$ (normal state) or at $40 \mathrm{~K}$ (CDW state). Note that during the STM measurements, the head of the microscope (and thus the tip) remains close to room temperature, which introduces a limitation of the system energy resolution. All experiments were performed with mechanically etched PtIr tips.

Interlayer magnetoresistance measurements have been performed at Laboratoire National des Champs Magnétiques Pulsés (LNCMP, Toulouse), up to $55 \mathrm{~T}$, using standard four wire method and low frequency current perpendicular to the 2D layers [13]. The data were recorded both during the steep increase of the field (corresponding to a time of approximatively $10 \mathrm{~ms}$ ) and during the exponential decay lasting roughly $100 \mathrm{~ms}$. The magnetic field was perpendicular to the sample platelet and therefore to the 2D layers. Data were collected for several temperatures between $1.6 \mathrm{~K}$ and $86 \mathrm{~K}$. 


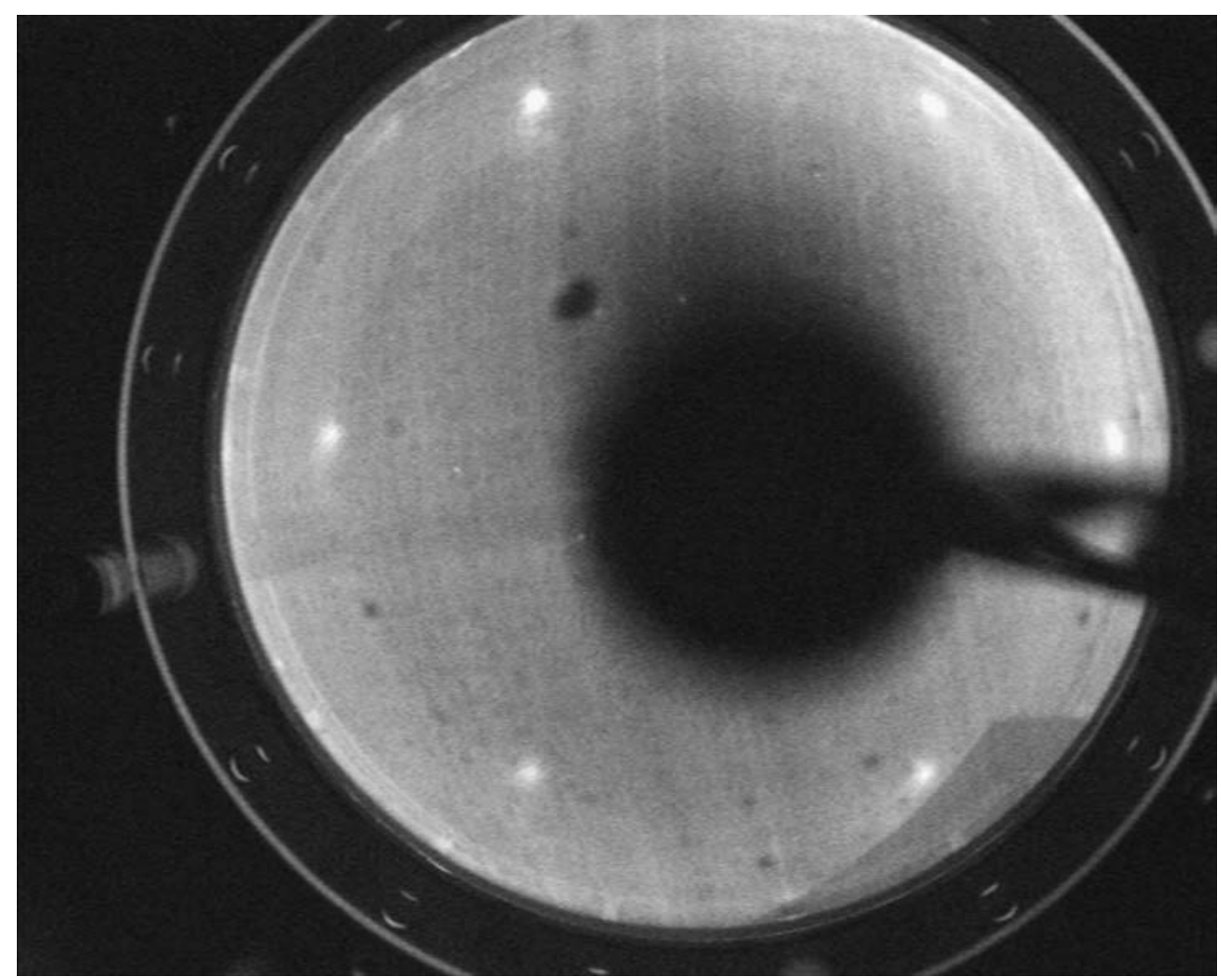

Fig. 3. Low Energy Electron Diffraction (LEED) pattern of the (001) plane of $\mathrm{KMo}_{6} \mathrm{O}_{17}$ at $20.5 \mathrm{eV}$.

\section{ARUPS studies of the band structure in the normal state}

The UPS spectrum obtained with the $\mathrm{He}_{\mathrm{II}}$ radiation at room temperature shows several structures that reveal a weak conduction band well separated from a valence band by a depleted zone of $1.5 \mathrm{eV}$. The fourfold structured valence band reflects the presence of distorted $\mathrm{MoO}_{6}$ octahedra in the crystallographic structure and extends over about $7 \mathrm{eV}$ down to the $\mathrm{K}_{3 \mathrm{p}}$ shadow core level. The occupied part of the conduction band has a bandwidth of $0.8 \mathrm{eV}$ (Fig. 4).

Such a band structure is very common to the metallic molybdenum oxides or tungsten bronzes [8]. As evidenced by resonant photoemission in $\eta-\mathrm{Mo}_{4} \mathrm{O}_{11}$, the parent compound of the molybdenum purple bronze, the conduction band is filled with Mo-4d type electrons, while the valence band has an oxygen character. Surface changes induced by aging in UHV, photon exposition or electron bombardment affect and hide the different features of the UPS spectra and progressively create states in the depleted zone between the conduction band and the valence band.

The determination of the band structure of the normal state has been the aim of ARUPS measurements performed at room temperature with the $\mathrm{He}_{\mathrm{I}}$ radiation. The spectra were collected along $\Gamma \mathrm{K}$ and $\Gamma \mathrm{M}$, the two main directions of the hexagonal first Brillouin zone (Fig. 5). The band structure was also analysed in the KM direction.

Dispersive structures are detected along the three directions: two bands cross the Fermi level along $\Gamma \mathrm{K}$, two along $\Gamma \mathrm{M}$ and one along $\mathrm{KM}$. In addition, one stationary band is

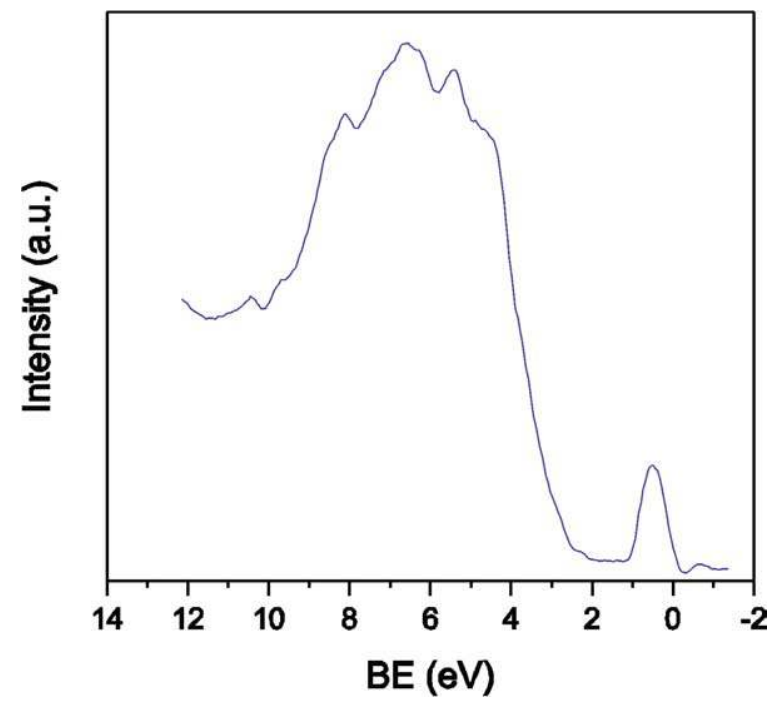

Fig. 4. Energy Distribution Curve (EDC) of $\mathrm{KMo}_{6} \mathrm{O}_{17}$ evidencing the valence and conduction bands.

detected in the $\Gamma \mathrm{M}$ direction at an energy close to $-0.5 \mathrm{eV}$ below the Fermi level. The bottom of the conduction band corresponds to the bottom of the lower dispersive structures and is very close to the stationary band (Fig. 6). These results are consistent with those obtained on $\mathrm{NaMo}_{6} \mathrm{O}_{17}$ [7], but deeply differ from the earliest results on $\mathrm{KMo}_{6} \mathrm{O}_{17}$ [15]. Our results corroborate the theoretical band structure calculated by M.-H. Whangbo et al. using a tight binding model [5]. For a better agreement, the theoretical energy should be rescaled by a factor close to 2.5 , which shifts the theoreti- 


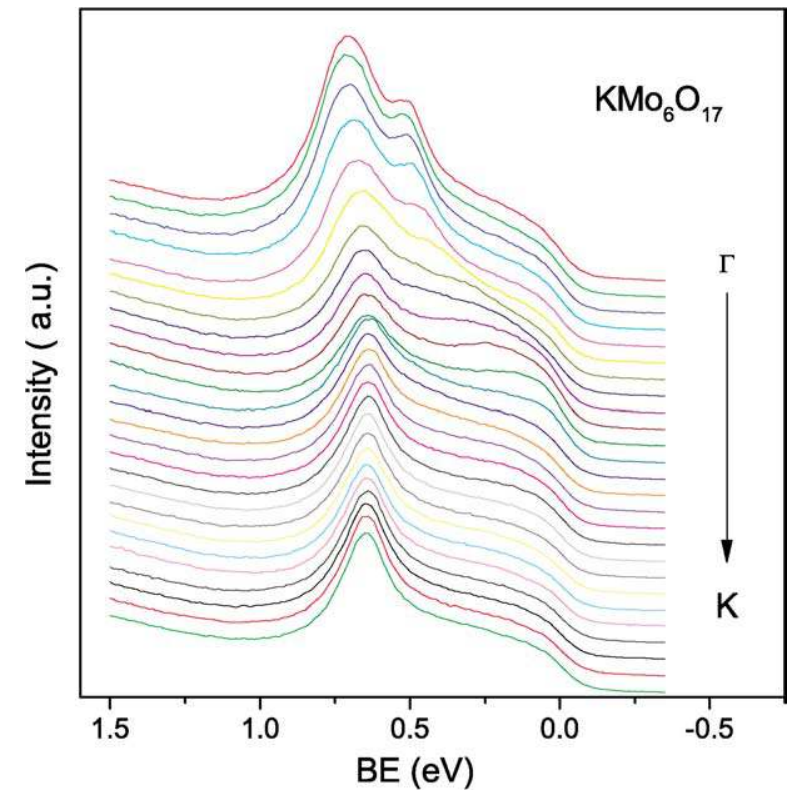

Fig. 5. Angle Resolved UV Photoemission Spectroscopy (ARUPS) spectra collected in the $\Gamma \mathrm{K}$ direction.

cal bandwidth to the experimental value of $0.8 \mathrm{eV}$. Nevertheless, the experimental results differ from the theoretical ones by two points: first, the bottom of the upper band, predicted to be at the midpoint of the conduction band, is actually shifted to lower energies. Secondly, the calculation predicts that three dispersive bands cross the Fermi level in the $\Gamma \mathrm{K}$ direction, while only two of them are detected. This discrepancy should be attributed to the experimental precision, since two of the three predicted bands are so close to each other that they cannot be resolved experimentally. In the $\Gamma \mathrm{M}$ direction, all three predicted bands are well detected.

The experimental results give the positions of five points of the $\mathrm{KMo}_{6} \mathrm{O}_{11}$ Fermi surface. These five points fit the calculated Fermi surface, described as a hexagonal inner sheet, a star-like outer sheet and six small pockets centred on the apexes of the hexagonal Brillouin zone [5]. They corroborate also the experimental Fermi surface obtained in Ref. [7].

Along the $\Gamma \mathrm{M}$ direction, the two points of the Fermi surface are found to be at $k_{1}=43.6 \% \Gamma \mathrm{M}$ and $k_{2}=56.7 \% \Gamma \mathrm{M}$. The difference between $k_{1}$ and $k_{2}, \Delta k_{\mathrm{F}}$, corresponds to the shortest distance between the inner and outer sheets. According to the hidden nesting model [5], these two sheets result from the superimposition of three pairs of planar Fermi surfaces parallel to the [100], [010] and [110] directions. These planes cross each other at points located on these three directions and $\Delta k_{\mathrm{F}}$ measures the distance between the sheets since the degeneracy at the crossing points is lifted by interchains couplings. Before lifting the degeneracy, the crossing point may be estimated at the position $\langle k\rangle=0.5\left(k_{1}+k_{2}\right)=$ $49.8 \%$ ГМ.

Since the charge density wave vector $\mathbf{q}$ in $\mathrm{KMo}_{6} \mathrm{O}_{17}$ points in the [100] direction, to satisfy the Peierls relation
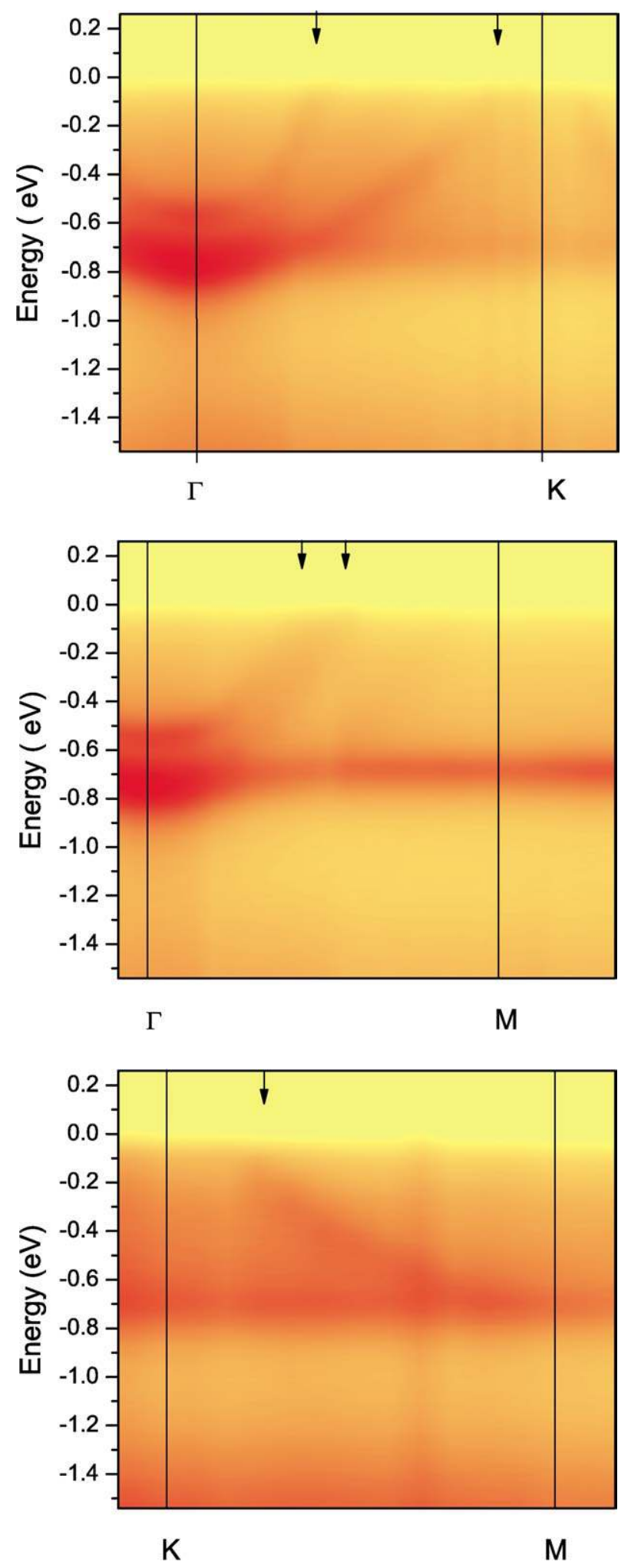

Fig. 6. Experimental band structure in the $\Gamma \mathrm{M}, \Gamma \mathrm{K}$ and $\mathrm{KM}$ directions. The arrows point out the positions of the bands crossing the Fermi level.

$q=2 k_{\mathrm{F}}$, the charge density wave vector $\mathbf{q}=0.5 \mathbf{a}^{*}[6,9]$ should be compared to $\langle 2 k\rangle=49 \% a^{*}$. Within the experimental accuracy, the Peierls relation is well satisfied. 


\section{STM imaging and spectroscopy of the charge density wave state}

We have performed STM measurements on the cleaved (001) $\mathrm{KMo}_{6} \mathrm{O}_{17}$ surface, at room temperature (normal metallic phase) and at $40 \mathrm{~K}$ (CDW metallic phase), on three different single crystals. Large scale images (not shown) exhibit atomic terraces of few hundred nanometers width, which confirm that cleavage in UHV is very efficient for this kind of material. Typical current-constant images with atomic resolution are shown in Fig. 7. For both images, the sample bias is fixed at $-450 \mathrm{mV}$, which implies that occupied states of the surface are probed. The inset in the lower-right part of the images is their corresponding 2D fast Fourier transform. At room temperature (Fig. 7(a)), a clear hexagonal atomic pattern with spacing $a=5.5 \pm 0.1 \AA$ is observed, in good agreement with the lattice parameter of the (001) plane found in the literature (5.538 $\AA$ ). We do not observe sublattice resolution on the image, and one can wonder which atomic plane is probed in Fig. 7. In such lamellar oxide compounds, it is commonly accepted that cleavage occurs between two adjacent $\mathrm{MoO}_{4}$ tetrahedra planes, which are weakly bonded. However, tight-binding calculations [5] indicate that electronic states with energy close to the Fermi level mainly come from the $4 \mathrm{~d}$ orbitals of molybdenum atoms located in the inner two Mo-O planes of the $\mathrm{Mo}_{6} \mathrm{O}_{17}$ slab. With a sample bias fixed at $-450 \mathrm{mV}$, it is likely that the images shown in Fig. 7 correspond to electronic states of this Mo-O plane, each bright spot being in coincidence with a single Mo atom of this plane. Although such a plane is not the topmost one, tunnelling into this plane probably occurs through one, two or three insulating planes participating to the barrier.

When the sample is cooled down to $40 \mathrm{~K}$, a striking feature is observed on constant-current images with atomic resolution, as shown in Fig. 7(b): a $2 \times 2$ superstructure is superimposed on the atomic pattern, leading to different apparent heights between adjacent atomic rows. This is very clear for the rows parallel to the direction given by the white arrow, for which one row over two appears $0.4 \AA$ higher. For the two other crystallographic directions (indicated by black arrows), it is somewhat more difficult to observe the superstructure, although it is truly present. One convincing point is to proceed to the 2D-Fast Fourier Transform of the image (see inset of Fig. 7(b)). The main features of this FFT are 6 outer peaks at $a^{*}=2 \pi / a$ due to the atomic lattice, and six inner peaks at $(0.50 \pm 0.05) a^{*}$, corresponding to the $2 \times 2$ superstructure.

This result is in good agreement with the commensurate $\mathrm{CDW}$ state of $\mathrm{KMo}_{6} \mathrm{O}_{17}$, which has been characterized previously by $\mathrm{X}$-ray diffuse scattering and electron diffraction studies [6]. A $(2 a, 2 b, c)$ Peierls lattice distortion has been observed, this corresponds to the $(2 \times 2)$ superstructure observed by STM. Our measurements are a direct probe of the CDW associated with the lattice distortion, which is still present in the conductive layer located underneath the sur-
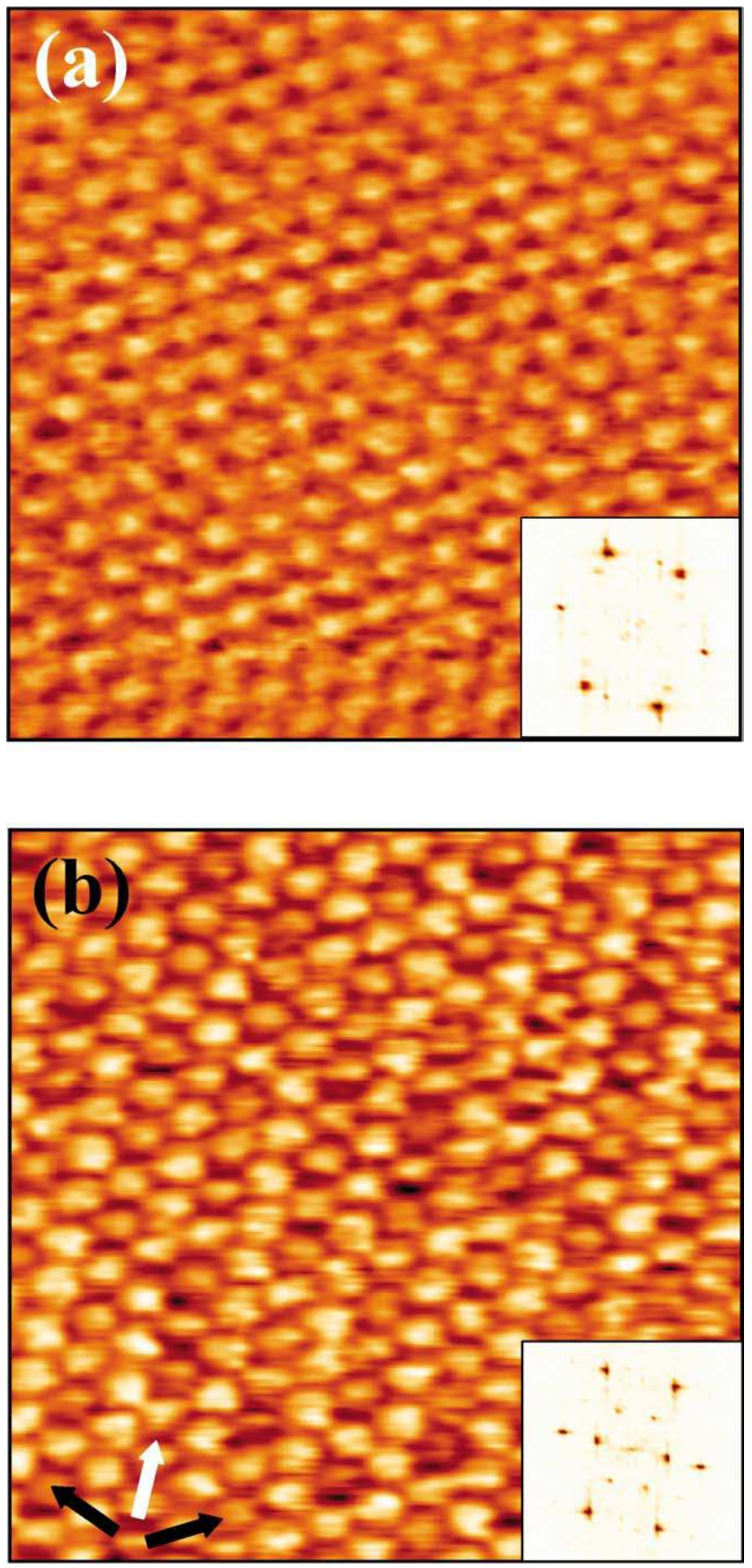

Fig. 7. $8 \times 8 \mathrm{~nm}^{2}$ constant current STM images of $\mathrm{KMo}_{6} \mathrm{O}_{17}$ cleaved in UHV. Sample bias is fixed at $-450 \mathrm{mV}$. On the lower-right hand we have plotted the corresponding 2D fast Fourier transform. (a) Normal phase image $(T=300 \mathrm{~K})$, showing the hexagonal atomic lattice. Tunnelling current: $150 \mathrm{pA}$. (b) CDW phase ( $T=40 \mathrm{~K}$ ). The quasi $2 \times 2 \mathrm{CDW}$ modulation is superimposed on the atomic pattern. Tunnelling current: $400 \mathrm{pA}$. The FFT shows the corresponding 6 peaks located at half the distance of the atomic lattice peaks.

face. To our knowledge, direct observation by STM of CDW on oxide layered compounds has only been reported by our group $[9,16]$. Obviously cleavage without UHV environment may explain the lack of results on this topic. 


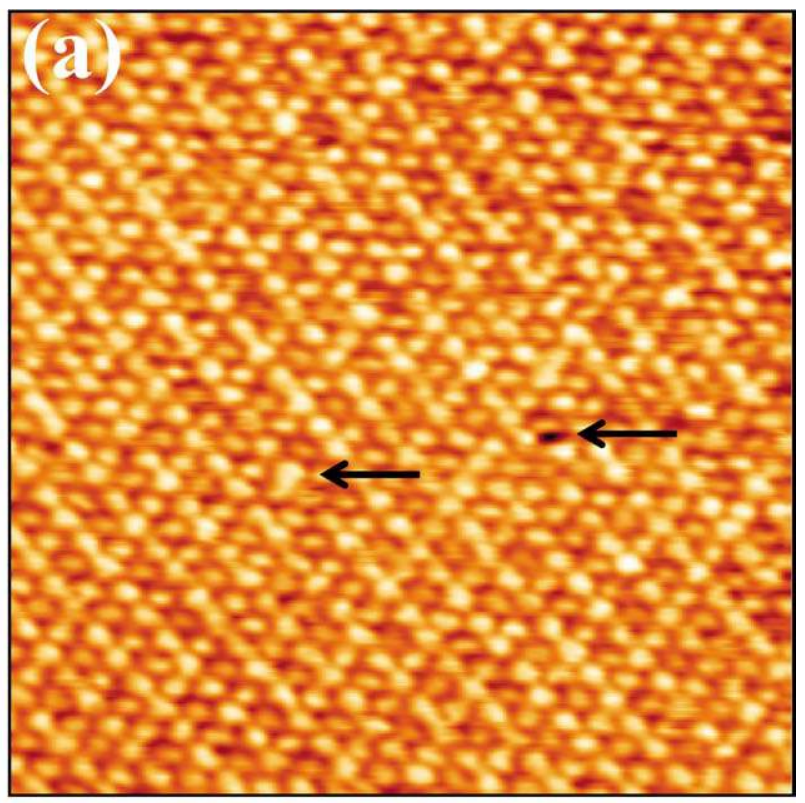

(b)

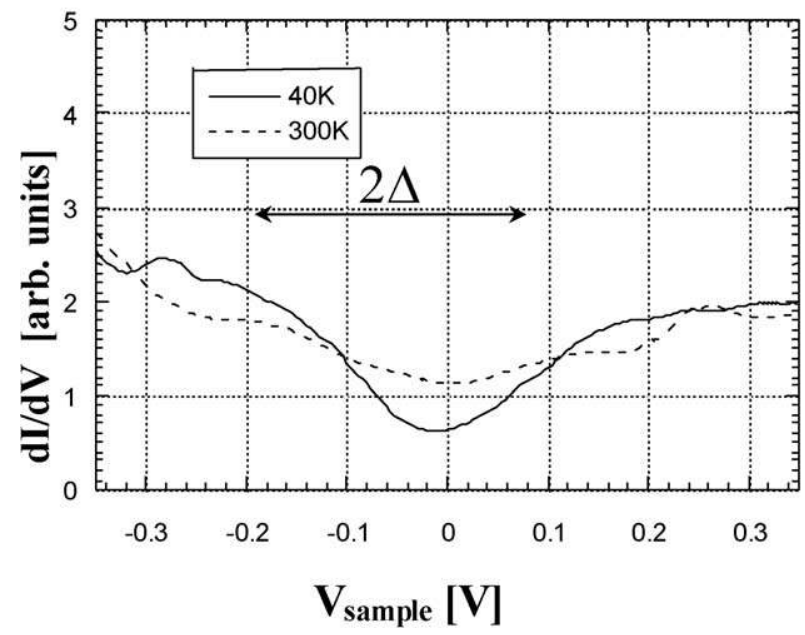

Fig. 8. (a) $13 \times 13 \mathrm{~nm}^{2}$ image of the $\mathrm{CDW}$ at $40 \mathrm{~K}$. Atomic surface defects are present (indicated by arrows), without any effect on the CDW arrangement. Sample bias: $-400 \mathrm{mV}$, Tunnelling current: $400 \mathrm{pA}$. (b) Scanning tunnelling spectroscopy measurements recorded at $40 \mathrm{~K}$ (solid line) and $300 \mathrm{~K}$ (dashed line). Each curve is an average of 64 numerical derivatives $I(V)$ spectra obtained in the open feedback loop.

Fig. 8(a) is a larger scale image of the CDW, showing that the presence of few atomic defects (indicated by arrows) does not perturbate the CDW arrangement. Large homogeneous terraces, as shown on Fig. 8(a), are suitable for spatially resolved tunneling spectroscopy. At low temperature, the dynamical conductance $d I / d V(\mathrm{~V})$ is proportional to the LDOS at energy $e V+E_{\mathrm{F}}$ ( $I$ is the tunnelling current, $V$ is the sample bias, $e$ is the electron charge and $E_{\mathrm{F}}$ the Fermi level). In the design of our STM the tip is not at low temperature, which increases the thermal broadening; however, an important dip in the conductance around the Fermi level has been measured at $40 \mathrm{~K}$. This is shown on
Fig. 8(b), on which we plotted the average of 64 curves obtained at $40 \mathrm{~K}$ (solid line curve), and at $300 \mathrm{~K}$ (dashed line). Although the apex of the tip probably changed between the two measurements, a decrease of the LDOS in an energy range of $2 \Delta=300 \pm 50 \mathrm{meV}$ was commonly observed at $40 \mathrm{~K}$ for the different samples. This value does not vary with the tip-sample distance, suggesting that we are not dealing with capacitive artefacts like Coulomb blockade. Since the tip remains at $300 \mathrm{~K}$, the thermal broadening of the LDOS structures is of the order of $120 \mathrm{meV}$ [9]. Thus the measured value $2 \Delta$ is probably larger than the real CDW gap. In spite of a large instrumental broadening one can measure a strong depression in the LDOS; therefore, the actual gap should be of the order of a few tens meV, i.e., of several $k T_{\mathrm{P}}$, as expected in a mean field model.

We ascribe the decrease of the dynamical conductance around zero bias to the diminution of the LDOS at $E_{\mathrm{F}}$ associated with the CDW state. As for other 2D systems with Peierls transition, part of the Fermi surface (FS) of $\mathrm{KMo}_{6} \mathrm{O}_{17}$ is destroyed in the CDW phase. For this particular compound, it has been shown that a large amount of conductive electrons is suppressed below $T_{\mathrm{P}}$, as evidenced by the resistivity measurements [6]. Because of the peculiar normal phase FS, which can be divided into three independent 1D sheets [17] (see ARUPS results described above), the opening of the gap below $T_{\mathrm{P}}$ leads to the suppression of large portions of the Fermi surface. This can be correlated to the large decrease (by a factor $\sim 2$ ) of the LDOS at $E_{\mathrm{F}}$ observed on our spectra. As mentioned previously, the CDW phase in $\mathrm{KMo}_{6} \mathrm{O}_{17}$ has been nicely described by the "1D hidden nesting" concept [5] and band structure calculations predict nesting wave vectors $\left(a^{*} / 2,0,0\right)$ and equivalent. These values are in excellent agreement with the wave vectors of the superstructure evidenced on the STM images (Fig. 7(b)).

In the 1D framework, it is expected that the phase of the CDW measured by STM is shifted by $\pi$ between occupied and empty states $[17,18]$. In a previous paper [9], we performed constant-current images at the same spot of the surface, at two opposite bias voltages. We observed a contrast reversal of the CDW superstructure (not shown), which we have attributed to a signature of the hidden 1D structure in the 2D Fermi surface of $\mathrm{KMo}_{6} \mathrm{O}_{17}$. Similar measurements made previously by one of the authors on the $2 \mathrm{D}$ metal $2 \mathrm{H}-$ $\mathrm{NbSe}_{2}$ did not lead to a simple contrast reversal [19].

\section{High magnetic field studies of the charge density wave state}

Fig. 9 shows the curves of the magnetoresistance $\Delta \rho / \rho_{0}$ vs $B$ at different temperatures. At the lowest temperature $(T=1.6 \mathrm{~K})$, they show a change of slope in the vicinity of $B_{1}=2 \mathrm{~T}$, a maximum at $B_{2}=9.4 \mathrm{~T}$ and two other maxima at $B_{3}=24.5 \mathrm{~T}$ and $B_{4}=31 \mathrm{~T}$. At higher temperatures, the two high field maxima merge into one which appears at a field $B_{3}$ varying between $27.5 \mathrm{~T}$ at $10 \mathrm{~K}$ to 


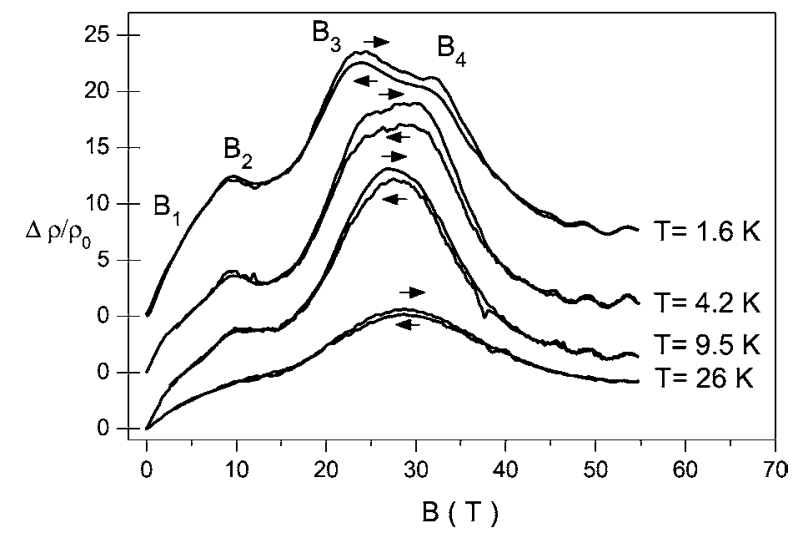

Fig. 9. Magnetoresistance $\Delta \rho / \rho$ vs $B$ for different temperatures. The curves corresponding to the increasing (upper trace) and decreasing (lower trace) field are shown.

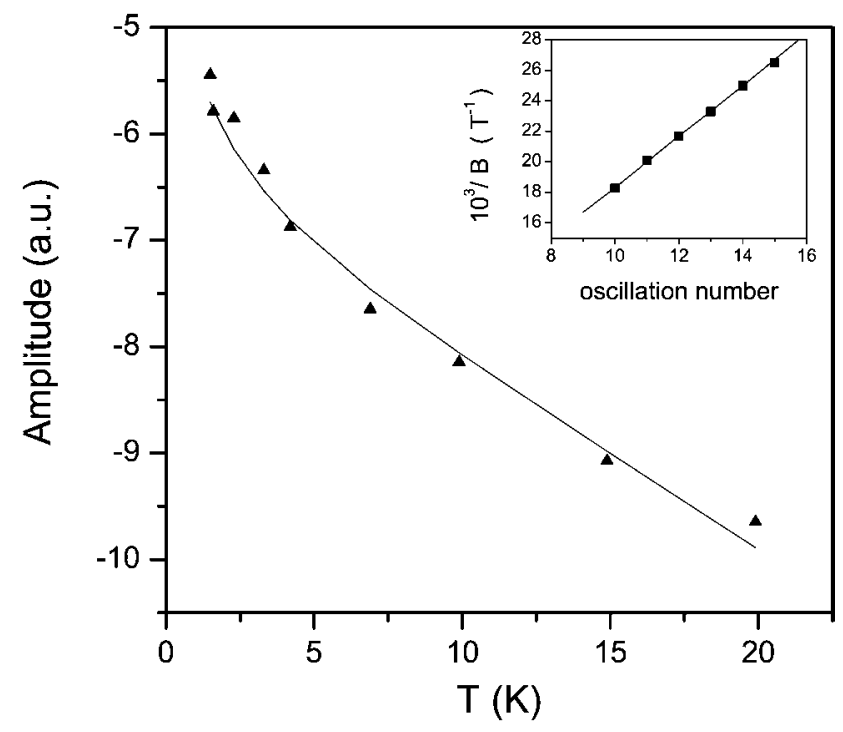

Fig. 10. Amplitude $A$ of the Shubnikov-de Haas oscillations vs temperature plotted as $\operatorname{Ln}(A / T)$ vs $T$. Insert: Reciprocal field $1 / B_{n}$ corresponding to the maxima of the high field oscillations vs an integer $n$.

$40 \mathrm{~T}$ at $86 \mathrm{~K}$. Above $B_{3}$ (or $B_{4}$ below $10 \mathrm{~K}$ ), oscillations of the Shubnikov-de Haas $(\mathrm{SdH})$ type appear clearly. Fig. 10 shows the analysis of these oscillations: the values of the inverse field $1 / B$ corresponding to the oscillation maxima are plotted as a function of integers found to vary between $n=10$ and $n=15$. The corresponding frequency of the oscillations, obtained from the Onsager relation $1 / B_{n}=(2 e / \hbar)\left(\pi / A_{\mathrm{F}}\right)(n+1 / 2)$ is $600 \pm 40 \mathrm{~T}$. The related area of the extremal orbit on the Fermi surface is found to be $5.4 \times 10^{-2} \AA^{-2}$. The amplitude $A$ of the oscillations is also plotted vs temperature in Fig. 9 as $\log (A / T)$ vs $T$. One may estimate a value for the cyclotron mass $m^{*}$ of $(0.6 \pm 0.1) m_{\mathrm{o}}$ where $m_{\mathrm{o}}$ is the free electron mass, from the relation $A \sim R_{\mathrm{t}}$ where $R_{\mathrm{t}}$ is the thermal factor: $R_{\mathrm{t}}=2 x e^{-x}$ and $x=2 p \pi^{2} m^{*} k T / m_{\mathrm{o}} e \hbar B$ ( $p$ is the order of the harmonic).

Fig. 11 shows the temperature of the anomalies plotted as a function of the fields $B_{1}, B_{2}, B_{3}$ and $B_{4}$. The low field

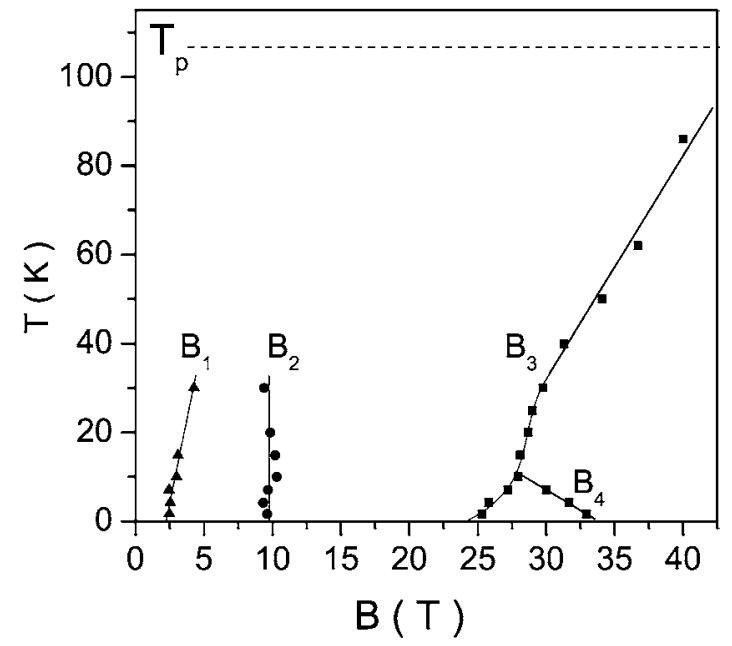

Fig. 11. Phase diagram showing on the horizontal scale the fields $B_{1}$ and $B_{2}$ of the anomalies and $B_{3}$ and $B_{4}$ of the transitions and on the vertical scale the corresponding temperatures.

anomalies $\left(B_{1}=2.5 \mathrm{~T}, B_{2}=10 \mathrm{~T}\right)$ do not seem to depend on temperature within the experimental accuracy. On the contrary, the fields $B_{3}$ and $B_{4}$ clearly depend on the temperature, the field $B_{3}$ approaching the Peierls temperature of $110 \mathrm{~K}$ at the highest fields.

One should first point out that the results reported here correspond to the low temperature state of $\mathrm{KMo}_{6} \mathrm{O}_{17}$. While the CDW state is established below $110 \mathrm{~K}$, one cannot exclude that the low temperature state is a mixed CDW/SDW state, as indicated by magnetic susceptibility measurements. Curves of the magnetization vs magnetic field show at low temperature a bending in the range of $2.5 \mathrm{~T}$ which could be consistent with an antiferromagnetic SDW behaviour [20]. In this context, it is likely that the change of slope found on the curves of the magnetoresistance at $B_{1}$ close to $2 \mathrm{~T}$ has the same origin. This could be related to a spin rotation away from the $c$-axis above $2 \mathrm{~T}$. Such a rotation corresponds to a change of sign of the magnetic susceptibility anisotropy, as indicated by magnetic torque data reported in Ref. [11]. The anomaly found at higher fields do not seem to be due to the de Haas-van Alphen oscillations. The maximum found at $B_{2}=10 \mathrm{~T}$ could be related to orbital effects induced by the magnetic field and improving the nesting. A new mixed CDW/SDW state could be stabilized above $B_{2}$. Such effects found in the (TMTSF) $)_{2} \mathrm{X}$ organic salts are associated to quantum nesting conditions and to first order transitions [21]. However specific heat measurements performed as a function of magnetic field at $T=2.5 \mathrm{~K}$ at the Grenoble High Magnetic Field Laboratory do not show first order transition at field $B_{2}$ [22].

The anomaly found at field $B_{3}$ and probably that found at field $B_{4}$ must be due to phase transitions. The presence of hysteresis at the $B_{3}$ transition indicates that this transition is first order. These properties are similar to those observed in the quasi-2D (BEDT-TTF) $)_{2} \mathrm{X}$ salts and are associated to the so-called kink transition [13,14,23]. 
The decrease of the magnetoresistance above $B_{3}$ and $B_{4}$ indicates that new CDW/SDW phases are stabilized above these fields. These phases have smaller CDW/SDW gaps than in the low field case. The new phase stabilized above $B_{3}$ (or $B_{4}$ below $10 \mathrm{~K}$ ) is characterized by Shubnikov-de Haas oscillations which correspond to an extremal area of the Fermi surface of $5.4 \times 10^{-2} \AA^{-2}$, therefore $3.6 \%$ of the 2D high temperature Brillouin zone (14.4\% of the low temperature one). This indicates the presence in this DW state of small cylindrical pockets induced by the gap openings.

These results suggest that the magnetic field above $B_{3}$ and $B_{4}$ closes partly the DW gaps. Such properties may be due to Pauli type effects taking over orbital effects above $30 \mathrm{~T}$. This is predicted by RPA calculations which show that in the case of quasi-1D systems, the DW state is destabilized by the Pauli effect [24]. Although these calculations are valid for $1 \mathrm{D}$ systems, the hidden nesting properties may justify the use of a 1D model for the present case. These effects depend on the ratio of the orbital to the Pauli coupling and on the ratio of the CDW coupling to the SDW one. Complex phase diagrams can be obtained depending on these parameters. Such a description could apply to $\mathrm{KMo}_{6} \mathrm{O}_{17}$. In this context, the line $B_{4}$ of this diagram could correspond to a variation of the CDW vector length $q$ as a function of the magnetic field and therefore to a CDW becoming incommensurate with increasing $B$.

However, all theoretical models predict that the energy associated to the critical magnetic field $\mu_{\mathrm{B}} B$ should be comparable to the thermal energy of the Peierls transition, $k_{\mathrm{B}} T_{\mathrm{P}}$, or to the Peierls gap [24]. This relation is obviously not observed in our case, since the Peierls temperature is above $100 \mathrm{~K}$ and the Peierls gap is found by STS to be in the range of a few ten meV. The Peierls temperature should be in the range of $10 \mathrm{~K}$ for these theoretical models to apply. One possible mechanism to solve this problem would be related to the complex 2D Fermi surface, which might include gaps much smaller than the average one. The magnetic field would then act on these special parts of the Fermi surface. Further experimental studies of the low temperature Fermi surface could corroborate such a model.

\section{Conclusion}

In this paper we examined recent results obtained on the quasi two-dimensional purple molybdenum bronze $\mathrm{KMo}_{6}-$ $\mathrm{O}_{17}$. Electron spectroscopy studies performed at room temperature in the normal metallic state corroborate the model of the hidden nesting, or hidden one-dimensionality, based on the existence in the crystal structure of infinite $\mathrm{MoO}_{6}$ octahedra chains along three crystallographic directions. This leads to three quasi-1D bands and to three planar Fermi surface sheets. Two of these bands are clearly observed by ARUPS. These studies also provide a value of the CDW nesting vector consistent with other studies, such as electron or X-ray diffraction in the CDW state.
STM studies established that the CDW state is a commensurate triple-q state and that no domain structure is associated with the three directions of q. X-ray or electron diffraction studies could not clarify this point. STS studies show a strong depression of the LDOS at the Fermi level below $T_{\mathrm{P}}$, consistent with the opening of a CDW gap of a few tens meV. This is roughly consistent with the order of magnitude of the Peierls temperature $\left(T_{\mathrm{P}}=110 \mathrm{~K}\right)$ in a mean field model. STM observation of contrast reversal indicates that the CDW phase is shifted by $\pi$ between occupied and empty states.

Finally, high magnetic field studies establish that, in the CDW state, two first order transitions take place above $25 \mathrm{~T}$ at $1.6 \mathrm{~K}$. These transitions correspond to partial closings of the CDW gap and are attributed to Pauli coupling effect which destroys the nesting conditions via Zeeman splitting of the bands. The high field transition corresponds probably to a variation of the CDW vector away from the commensurate value. However, theoretical studies would predict transition in fields one order of magnitude larger than the observed values. This is a puzzling problem which might be related to the complexity of the real Fermi surface. Experimental and theoretical Fermi surface studies are necessary to solve this problem.

\section{Acknowledgements}

The work on $\mathrm{KMo}_{6} \mathrm{O}_{17}$ would not have been possible without the initial crystal structure studies performed by H. Vincent et al. more than 20 years ago at Laboratoire de Cristallographie, CNRS Grenoble, under the leadership of E.F. Bertaut.

The authors wish to thank E. Hannappel and C. Proust for their help during the high magnetic field studies at LNCMP and V. Mineev, I. Wagner and D. Zanchi for helpful discussions.

\section{References}

[1] C. Schlenker (Ed.), Low Dimensional Properties of Molybdenum Bronzes and Oxides, Kluwer, Dordrecht/Norwell, MA, 1989.

[2] Oxide bronzes, in: M. Greenblatt (Ed.), Int. J. Mod. Phys. B 7 (1993) 4045.

[3] C. Schlenker, J. Dumas, M. Greenblatt, S. van Smaalen (Eds.), Physics and Chemistry of Low-Dimensional Inorganic Conductors, NATO ASI Series, Series B: Physics, vol. 354, Plenum, New York, 1996.

[4] H. Vincent, M. Ghedira, J. Marcus, J. Mercier, C. Schlenker, J. Sol. State Chem. 47 (1983) 113.

[5] M.H. Whangbo, E. Canadell, C. Schlenker, J. Amer. Chem. Soc. 109 (1987) 6308;

M.H. Whangbo, E. Canadell, P. Foury, J.P. Pouget, Science 252 (1991) 96;

E. Canadell, M.H. Whangbo, Oxide bronzes, in: M. Greenblatt (Ed.), Int. J. Mod. Phys. B 7 (1993) 4005;

E. Canadell, Chem. Mater. 10 (1998) 2770.

[6] C. Escribe-Filippini, K. Konate, J. Marcus, C. Schlenker, R. Almairac, R. Ayroles, C. Roucau, Phil. Mag. B 50 (1984) 321. 
[7] G.H. Gweon, J.W. Allen, J.A. Clack, Y.X. Zhang, D.M. Poirier, P.J. Benning, Olson, J. Marcus, C. Schlenker, Phys. Rev. B 55 (1997) R13353.

[8] H. Guyot, H. Balaska, J. Marcus, J. Phys. IV 12 (2002) 9-95.

[9] P. Mallet, K.M. Zimmermann, Ph. Chevalier, J. Marcus, J.Y. Veuillen, J.M. Gomez Rodriguez, Phys. Rev. B 60 (1999) 2122; J.Y. Veuillen, P. Mallet, L. Magaud, S. Pons, J. Phys.: Condens. Matter. 15 (2003) S2547.

[10] A. Rötger, C. Schlenker, J. Dumas, J. Marcus, S. Dubois, A. Audouard, L. Brossard, J.P. Ulmet, S. Askenazy, Synth. Metals 55-57 (1993) 2725.

[11] J. Dumas, H. Guyot, H. Balaska, J. Marcus, D. Vignolles, I. Sheikin, A. Audouard, L. Brossard, C. Schlenker, Physica B 346-347 (2004) 314.

[12] M. Tian, S. Yue, J. Shi, S. Li, Y. Zhang, J. Phys. Cond. Mat. 13 (2001) 311 ;

M. Tian, S. Yue, S. Li, Y. Zhang, J. Shi, J. Appl. Phys. 89 (2001) 3408; M. Tian, S. Yue, Y. Zhang, Phys. Rev. B 65 (2002) 104421.

[13] C. Proust, A. Audouard, A. Kovalev, D. Vignolles, M. Kartsovnik, L. Brossard, N. Kushch, Phys. Rev. B 62 (2000) 2388.
[14] D. Vignolles, A. Audouard, L. Brossard, S. Pesotskii, R. Lyubovskii, M. Nardone, E. Haanappel, R. Lyubovskaya, Eur. Phys. J. B 31 (2003) 53.

[15] K. Ohtake, H. Matsuoka, R. Yamamoto, M. Doyama, H. Sakamoto, T. Mori, K. Soda, S. Suga, J. Phys. C 19 (1986) 7207.

[16] P. Mallet, H. Guyot, J.Y. Veuillen, N. Motta, Phys. Rev. B 63 (2001) 165428.

[17] M.H. Whangbo, J. Chem. Phys. 73 (1980) 3854.

[18] J. Tersoff, Phys. Rev. Lett. 57 (1986) 440.

[19] P. Mallet, W. Sacks, D. Roditchev, D. Défourneau, J. Klein, J. Vac. Sci. Technol. B 14 (1996) 1070.

[20] J. Dumas, E. Bervas, J. Marcus, D. Salomon, C. Schlenker, G. Fillion, J. Magn. Magn. Mat. 31 (1983) 535.

[21] M. Héritier, G. Montambaux, P. Lederer, J. Phys. Lett. 45 (1984) L-94; G. Montambaux, M. Héritier, P. Lederer, Phys. Rev. Lett. 55 (1985) 2078.

[22] J. Dumas, H. Guyot, J. Marcus, C. Schlenker, I. Sheikin, unpublished.

[23] T. Osada, R. Yagi, A. Kawasumi, S. Kagoshima, N. Miura, M. Oshima, G. Saito, Phys. Rev. B 41 (1990) 5428.

[24] D. Zanchi, A. Bjelis, G. Montambaux, Phys. Rev. B 53 (1996) 1240. 